\title{
Living donor liver transplantation in an adult patient with situs inversus totalis
}

Yücel Yankol, Nesimi Mecit, Turan Kanmaz, Koray Acarlı, Münci Kalayoğlu

\section{ABSTRACT}

Organ Transplantation Center, Memorial Şişli Hospital, İstanbul, Turkey

Address for Correspondence Yücel Yankol

e-mail:yyankol@yahoo.com

Received: 24.12.2014

Accepted: 18.01.2015

Available Online Date: 06.07.2015

CCopyright 2015

by Turkish Surgical Association

Situs inversus totalis (SIT) is a rare congenital anomaly, and liver transplantation (LT) in an adult SIT patient is extremely rare. Liver transplantation in a SIT patient is also technically challenging due to reversed anatomical structures. Here we present the case of an 18-year-old female with SIT in whom left lobe living donor LT was performed. The patient suffered from cirrhosis due to autoimmune hepatitis. The recipient and donor are doing well without complications 20 months after LT. Situs inversus totalis should not be considered a contraindication for LT. If possible, use of a living donor left lobe graft for LT is more feasible than a living donor right lobe graft. It is also technically easier than using deceased donor full-size liver graft in SIT patients who require liver transplantation.

Keywords: Situs inversus totalis, liver transplantation, living donor

\section{INTRODUCTION}

Situs inversus totalis (SIT) is a rare congenital anatomic variation characterized by a mirror-image orientation of abdominal and thoracic viscera relative to the midline compared with normal anatomy. The incidence of SIT is estimated to be 1 in 15,000-20,000 births and its cause is currently unknown. In some cases, SIT is associated with other anomalies such as biliary atresia, visceral vascular anomaly, and polysplenia. Situs inversus totalis causes no problems for many people during their life span; however, it can be a serious problem when a patient with SIT requires liver transplantation (LT). Previously, SIT was regarded as an absolute contraindication for LT. The asymmetric structure causes technical difficulties during the positioning of the graft and vascular anastomosis (1-3).

Biliary atresia (BA) is the most common indication for LT in SIT patients because $10 \%-20 \%$ of SIT patients are also born with BA. As a result of this rare disorder, most LT cases in SIT patients are in the pediatric population, and LT in adult SIT patients is extremely rare. There are few adult cases reported in the literature with discussion of technical considerations of using living donor right vs. left lobes (2-4). Here we present the case of living donor LT in an adult SIT patient using a left lobe graft with a successful result, with no donor complication. We also discuss the technical advantage of using the left lobe graft.

\section{CASE PRESENTATION}

An 18-year-old female with cirrhosis secondary to autoimmune hepatitis was referred for evaluation and treatment. Her body weight was $53 \mathrm{~kg}$ and model for end-stage liver disease score was 28. Pretransplant three-dimensional abdominal computed tomography (CT) scan confirmed SIT with a cirrhotic liver, minimal ascites, and splenomegaly (Figure 1a, b). Thoracic X-ray imaging also revealed dextrocardia (Figure 1c). The patient's donor was her cousin, a 35-year-old female with suitable right and left lobe hepatic volumes. The donor's body weight was $72 \mathrm{~kg}$ and body-mass index was 26 . Preoperative donor CT scan revealed normal anatomy of the portal and hepatic venous system with an additional artery originating from the right hepatic artery, supplying segment 4 of the left lobe (Figures 2a, b). Total liver volume was $1.354 \mathrm{~mL}$ on preoperative CT volume measurement. Right liver lobe volume was $810 \mathrm{~mL}$ (59.8\% of total liver volume) and left liver lobe volume was $544 \mathrm{~mL}$ ( $40.2 \%$ of total liver volume). Preoperative measurement of the left lobe to recipient weight ratio (GRWR) was 1.04\%. Preoperative magnetic resonance cholangiopancreatography (MRCP) revealed right lobe anterior sector and posterior sector bile ducts opening separately to the common bile duct, with one left lobe bile duct; this was confirmed on perioperative cholangiography (Figure $2 \mathrm{c}$ ).

After reviewing the donor liver studies, we decided to use the donor left lobe for transplantation. The donor surgery, a left hepatic lobectomy including the middle hepatic vein to the left hepatic lobe, was performed without complication (Figure $2 \mathrm{~d}$ ). At the end of the surgery, as expected, there was one bile duct, one portal vein, one hepatic vein orifice including left and middle hepatic veins, and two arteries with the left lobe graft (Figure 3a). Actual graft weight was $490 \mathrm{~g}$ and actual GRWR was $0.93 \%$. Recipient hepatectomy was completed uneventfully, with the donor left lobe graft placed in the left subphrenic 


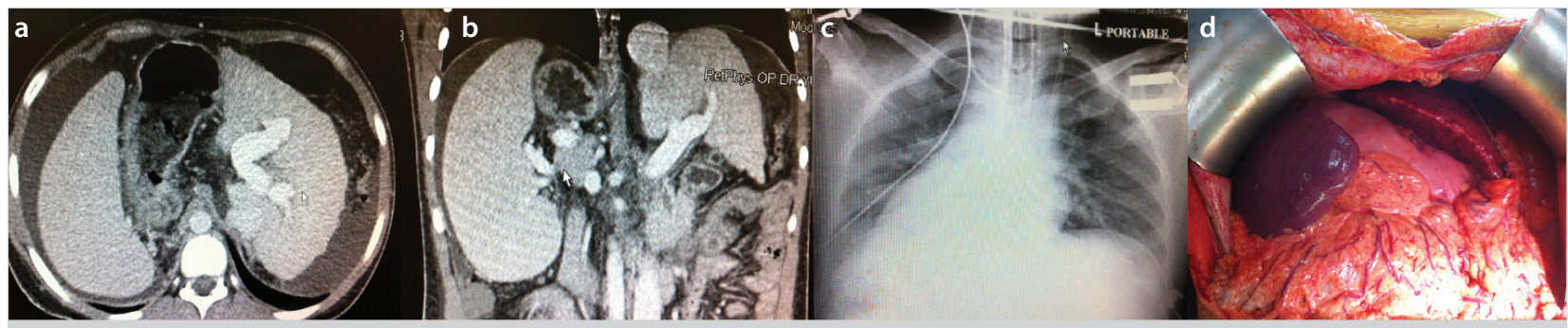

Figure 1. a-d. Preoperative and perioperative findings of the recipient. (a) Preoperative horizontal CT image showing SIT. (b) Preoperative coronal CT image showing SIT. (c) Preoperative thorax X-ray image showing dextrocardia. (d) Perioperative abdominal view of the recipient showing SIT

CT: computed tomography; SIT: situs inversus totalis

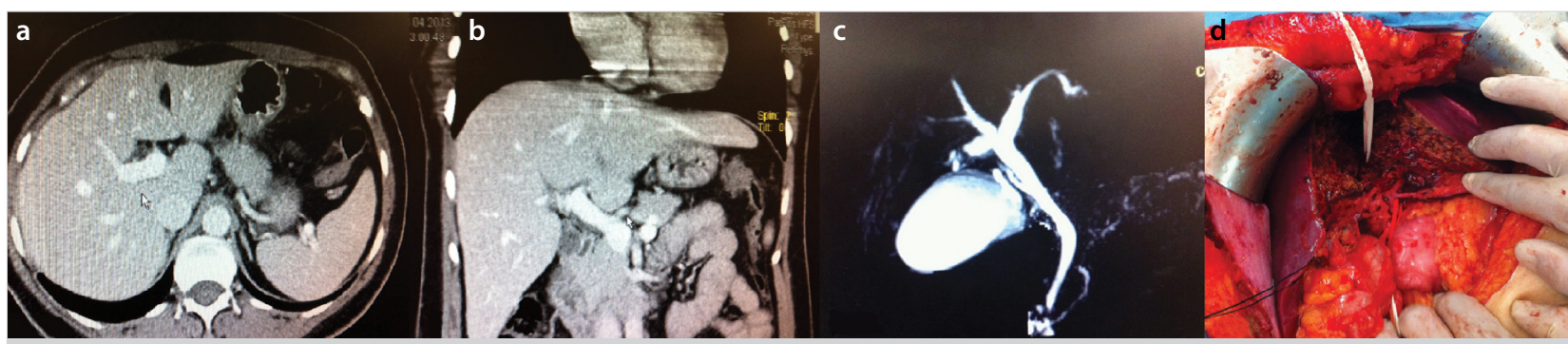

Figure 2. a-d. Preoperative and perioperative findings of the donor. (a) Preoperative horizontal CT image showing normal anatomy. (b) Preoperative coronal CT image showing normal anatomy. (c) Preoperative MRCP image. (d) Perioperative abdominal view of the donor

CT: computed tomography; MRCP: magnetic resonance cholangiopancreatography

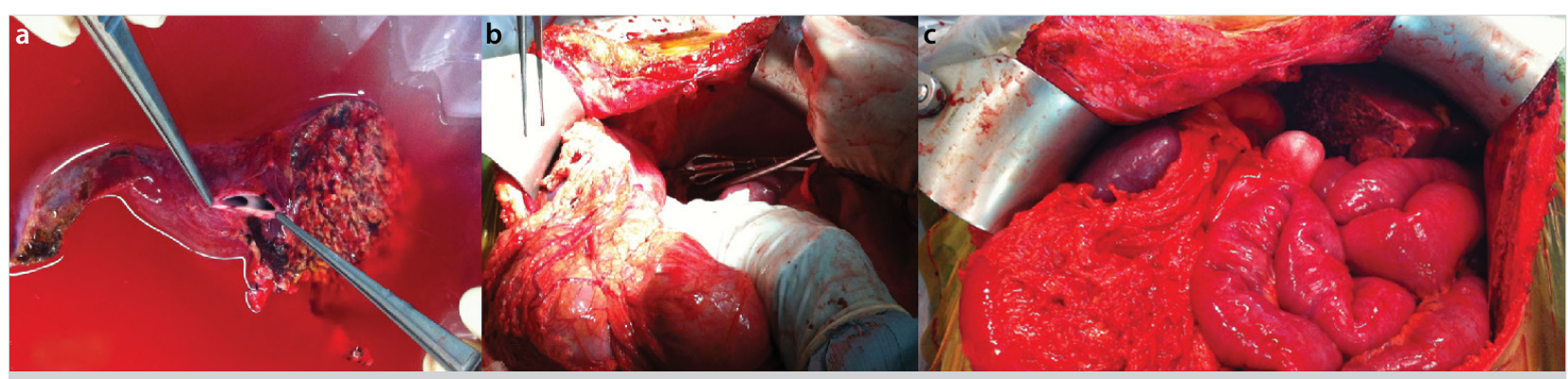

Figure 3. a-c. Perioperative findings of the recipient and the graft (a). Perioperative back-table view of the graft showing middle and left orifices (b). Perioperative abdominal view of the recipient after hepatectomy (c). Perioperative abdominal view of the recipient after LT

space. The triangulated middle and left hepatic vein trunk of the left lobe graft was anastomosed to the triangulated recipient combined left and middle hepatic vein orifices. The graft portal vein was anastomosed to the recipient left portal vein. Two separate graft hepatic arteries were anastomosed to the recipient left and right hepatic arteries under a microscope using a microsurgery technique. The bile duct of the left lobe graft was anastomosed to the recipient common bile duct without complication, with the advantage of using a left lobe graft in this SIT recipient (Figures 3b, c). Cold ischemia time was $75 \mathrm{~min}$ and warm ischemia time was $30 \mathrm{~min}$. Nine units of red blood cell products and 13 units of fresh frozen plasma were used during the surgery. An intraoperative Doppler ultrasound confirmed blood flow in all vessels of the left lobe hepatic graft.

The donor's postoperative course was excellent and she was discharged on postoperative day 7 . The recipient was extubated in the operating room and stayed in the intensive care unit for 1 day. Six days post LT, the recipient's liver enzyme levels began to increase and she was treated for acute rejection with cortisone bolus therapy. She was discharged 14 days post LT. Written informed consent was obtained from the patient. Both donor and recipient are currently doing well without any complications 20 months after surgery.

\section{DISCUSSION}

Situs inversus totalis, a rare condition of abnormal visceral rotation, was first described in animals by Aristotle (5). Fabricius reported the first SIT case in a human in the year $1600(5,6)$. Then, the cause of SIT was unknown, but chromosomes involved in lateralization and polarity were implicated (7). Situs inversus totalis continues to be interesting and noteworthy in the surgical realm due to the technical challenges involved in surgical treatment for SIT (5).

The first successful LT for SIT was performed by Raynor et al. (8) from a deceased donor. Since then, LT has been continually performed in SIT patients, most often in pediatric recipients. Over time, it has been determined that although the 
procedure is technically challenging due to necessary operative modifications, with comparable outcomes to LT for other diagnoses, SIT is not a contraindication to LT. Following several successful small series of LT in pediatric SIT patients using living donor left lateral segment grafts as well as adult cases using whole deceased donor grafts, the first successful adult living donor right lobe LT in an SIT recipient was performed in 2004 by Heimbach et al. (4), followed by the first successful adult living donor left lobe LT in an SIT recipient in 2006 by Soejima et al. (2). Since then, only a few cases of LT from a living donor to an adult recipient with SIT have been reported. A few case reports have indicated successful results in LT grafts from living donors with SIT in recipients with normal anatomy (9).

In SIT patients, the orientation and position of the hepatic vessels is reversed and the right subphrenic space contains spleen and stomach. The main problem this poses for LT is graft positioning due to the mismatch between the graft and recipient left upper quadrant space. The literature describes limited experience with successful techniques for resolution of these problems when performing LT in a recipient with SIT. The most common technique is midline positioning or left shifting, with or without clockwise rotation of the graft. This may cause vena cava compression due to the large right lobe of the graft $(1,3)$. To solve this problem, Rayhill et al. (10) suggested using a technical trick of a $180^{\circ}$ flip-over implantation (facing backward) of the whole liver graft into the recipient's left subphrenic space, allowing good arrangement of hilar structures. This technique was also successfully used by Chun et al. (9) in transplanting a living donor right lobe from an SIT donor to a recipient with normal anatomy. Chun et al. (9) suggested using high hilar dissection and long hepatic vein cuff preparation. In addition, Kim et al. (3) reported a successful result in an SIT recipient by using a living donor right posterior section graft with reversed placement technique.

Living donor LT differs from deceased donor LT, as living donor grafts always have short vessels and bile ducts, which require different recipient hepatectomy techniques, sometimes necessitating vessel reconstruction. One advantage of living donor LT is the opportunity for careful preoperative evaluation of both donor and recipient anatomy. This allows for preoperative planning to prevent technical problems. During the recipient hepatectomy, importance is placed on high hilar dissection and long hilar structures for a long hepatic vein cuff. Additional vascular graft reconstruction may be required while performing the anastomosis between donor and recipient vessels. Heimbach et al. (4) suggest mobilizing the recipient omentum and ascending colon to fill the empty hepatic fossa. Along with the plication of the diaphragm, this may provide satisfactory support to the liver graft.

In adult patients, a left lobe liver graft rarely provides sufficient liver volume. In the present case of a living donor left lobe, the only technical trick used was the rotation of the graft slightly to the left to accommodate the hepatic vein anastomosis. In addition, a triangulated suture technique was used during the hepatic vein anastomosis. This is the preferred technique by our group because we believe that this technique prevents torsion and is the best to increase the highly important venous outflow of the graft.

\section{CONCLUSION}

Liver transplantation in SIT patient can be performed with successful results using a living or deceased donor graft. Different technical tricks allow for successful results. In addition, liver grafts from both deceased and living donors with SIT can be used for recipients with normal anatomy or with SIT when certain technical tricks are performed. If possible, using a left lobe graft from a living donor is more feasible than using a right lobe graft in adult SIT recipients for donor safety and technical advantages because it is less technically challenging than using a full-sized liver graft.

Informed Consent: Written informed consent was obtained from patient who participated in this case.

Peer-review: Externally peer-reviewed.

Author Contributions: Concept - Y.Y., K.A.; Design - Y.Y.; Supervision - M.K., K.A.; Funding - M.K., K.A.; Materials - Y.Y., K.A.; Data Collection and/or Processing - Y.Y., N.M.; Analysis and/or Interpretation - Y.Y., T.K., K.A.; Literature Review - Y.Y., T.K.; Writer - Y.Y., K.A., M.K.; Critical Review - K.A., M.A., T.K., N.M.; Other - Y.Y., N.M.

Conflict of Interest: No conflict of interest was declared by the authors.

Financial Disclosure: The authors declared that this study has received no financial support.

\section{REFERENCES}

1. Kamei H, Onishi Y, Ogawa K, Uemoto S, Ogura Y. Living donor liver transplantation using a right liver graft with additional vein reconstructions for patient with situs inversus. Am J Transplant 2014; 14: 1453-1458. [CrossRef]

2. Soejima $Y$, Meguro $M$, Taketomi A, Ikegami T, Yamashita $Y$, Harada $\mathrm{N}$, et al. Left lobe living donor liver transplantation in an adult patient with situs inversus: technical considerations. Transpl Int 2008; 21: 384-389. [CrossRef]

3. Kim BW, Bae BK, Xu W, Wang HJ, Kim MW. Living donor liver transplantation for an adult patient with situs inversus totalis. World J Gastroenterol 2010; 16: 2311-2313. [CrossRef]

4. Heimbach JK, Menon KV, Ishitani MB, Nyberg SL, Jankowski CJ, Lindor KD, et al. Living donor liver transplantation using a right lobe graft in an adult with situs inversus. Liver Transpl 2005; 11: 111-113. [CrossRef]

5. Nursal TZ, Baykal A, Iret D, Aran O. Laparoscopic cholecystectomy in a patient with situs inversus totalis. J Laparoendosc Adv Surg Tech A 2001; 11: 239-241. [CrossRef]

6. Mayo CW, Rice RG. Situs inversus totalis; a statistical review of data on 76 cases with special reference to disease of the biliary tract. Arch Surg 1949; 58: 724-730. [CrossRef]

7. Aylsworth AS. Clinical aspects of defects in the determination of laterality. Am J Med Genet 2001; 101: 345-355. [CrossRef]

8. Raynor SC, Wood RP, Spanta AD, Shaw BW. Liver transplantation in a patient with abdominal situs inversus. Transplantation 1988; 45: 661663. [CrossRef]

9. Chun JM, Jung GO, Choi GS, Park JB, Kwon CH, Kim SJ, et al. Living donor liver transplantation using a graft from a donor with situs inversus totalis. Liver Transpl 2009; 15: 666-669. [CrossRef]

10. Rayhill SC, Scott D, Orloff S, Horn JL, Schwartz J, Zaman A, et al. Orthotopic, but reversed implantation of the liver allograft in situs inversus totalis-a simple new approach to a difficult problem. Am J Transplant 2009; 9: 1602-1606. [CrossRef] 